\title{
APLICAÇÃO WEB PARA OBTENÇÃO DE PARÂMETROS DO CIRCUITO EQUIVALENTE DE TRANSFORMADORES MONOFÁSICOS
}

DOI: 10.37702/2175-957X.COBENGE.2021.3415

Natã Rodrigo Santos Pereira - nata.rodrigo.p@gmail.com

Faculdades Metropolitanas Unidas

Rua Frederick Delius 6

04855-240 - São Paulo - SP

Victoria Alejandra Salazar Herrera - victoria.herrera@fmu.br

Faculdades Metropolitanas Unidas

Praça Rui Barbosa 72

09210-620 - Santo André - SP

Resumo: O objetivo deste trabalho foi desenvolver uma aplicação web para calcular os valores das resistências e reatâncias do circuito equivalente do transformador monofásico. Esta aplicação web caracterizada como objeto de aprendizagem foi elaborada em em HTML, CSS e JavaScript, apresentando resultados numéricos e gráficos para cinco tipos de circuito equivalente do transformador. Este OA surgiu como proposta de melhoria a um OA semelhante elaborado em Microsoft Excel o qual presentava como principal desvantagem a necessidade de usar computador e ter instalado o Microsoft Excel. Além de melhorar as características de durabilidade e interoperabilidade do OA inicial, verificou-se que a nova interface foi mais amigável aos usuários. Ambos OAs foram aplicados na mesma turma de estudantes, em períodos letivos diferentes, para avaliação de usabilidade, mas se foram atingidos os objetivos pedagógicos ainda precisa ser verificado. Finalmente apresentam-se sugestões de uso deste OA de forma diferenciada para docente e para estudante.

Palavras-chave: Aplicação web, Objeto de Aprendizagem, Transformador monofásico, Circuito equivalente 


\section{APLICAÇÃO WEB PARA OBTENÇÃO DE PARÂMETROS DO CIRCUITO EQUIVALENTE DE TRANSFORMADORES MONOFÁSICOS}

\section{INTRODUÇÃO}

O circuito equivalente de equipamentos elétricos é uma ferramenta utilizada para o estudo das máquinas elétricas em regime permanente, simplificando as equações que descrevem o comportamento das máquinas à forma algébrica, representando resistências, indutâncias, tensões e correntes mediante fasores. Uma desvantagem do uso desta ferramenta, é o grau de abstração que se requer para o entendimento da mesma, pois como todo modelo, os elementos presentes no circuito não necessariamente existem no equipamento físico.

A capacidade de abstração, fácil uso de ferramentas matemáticas e solução de problemas são geralmente característica dos estudantes de engenharia, porém deve ser considerar que cada indivíduo tem um estilo diferente de aprendizagem, como afirmado por Nafea et al. (2019) alguns estudantes tem preferência por realizar tarefas e discutir sobre conceitos, outros preferem manipular e examinar a informação. Visto este perfil diferenciado de cada estudante verificou-se em sala de aula a dificuldades para a correta obtenção do circuito equivalente do transformador monofásico.

Uma vez levantada esta dificuldade, diversas estratégias foram adotadas, como uso de infográficos, uso de simuladores e experiências em laboratório. Mas considera-se que o tempo usado para o ensinamento deste assunto, limitado pela duração da aula, também é um fator que dificulta o aprendizado, surgindo assim a ideia de elaborar um Objeto de Aprendizagem (OA) para que o aluno consiga verificar a correta obtenção dos parâmetros do circuito equivalente.

Os "OAs fornecem um suporte no processo de aprendizado, e são de grande valia principalmente quando o assunto a ser estudado é complexo”, Herrera et al. (2020). Considerando como OA "todo objeto digital que possa ser reutilizado ou referenciado na aprendizagem apoiada pela tecnologia" Braga (2014), realizou-se uma verificação de OAs existentes para o estudo de transformadores considerando principalmente websites e simulações.

Entre os websites consideram-se relevantes ACADEMY (2012) com diversos OAs para o estudo eletromagnetismo e máquinas elétricas mas o qual não considera o circuito equivalente do transformador; HOLDINGS (2016) que apresenta um site interativo para analisar o comportamento das tensões em função do número de espiras.

Quanto a simuladores foram encontrados trabalhos realizados no ambiente computacional MATLABß/Simulink Ayasun; Nwankpa (2006) e Koochaki (2015), ambos realizam os ensaios clássicos para o obtenção dos parâmetros do transformador e apresentam excelentes resultados quando comparados com resultados obtidos em laboratório, porém para seu correto funcionamento é necessário já contar com o set de parâmetros do transformador. Lopes et al. (2020), apresenta uma simulação do transformador com elementos finitos com objetivos semelhantes aos dois trabalhos descritos anteriormente, mas com a vantagem de apresentar também uma interface gráfica do transformador semelhante a como ele é fisicamente. Estas simulações podem substituir a ausência de equipamentos de laboratório ou ser aplicados em um curso EaD, mas o aluno ainda precisa construir o circuito equivalente. Finalmente, Herrera et al. (2020) apresenta um OA que pode ser executado no programa Microsoft Excel, que realiza os cálculos para obtenção dos circuitos equivalentes (completo, $T$ e $L$ referidos a ambos lados do 
transformador) e tem uma interface gráfica com os valores dos parâmetros para cada circuito, porém é necessário o uso do computador e, obviamente, ter instalado o Microsoft Excel.

O presente trabalho tem como objetivo aprimorar o trabalho realizado por Herrera et al. (2020), elaborando uma aplicação web para calcular os valores dos parâmetros do circuito equivalente do transformador, facilitando assim o uso do mesmo em diferentes dispositivos, garantindo assim que os estudantes possam usá-lo também em dispositivos móveis.

$\mathrm{Na}$ seção 2 deste trabalho apresenta-se uma breve descrição dos circuitos equivalentes do transformador, na seção 3 descreve-se a metodologia usada para 0 desenvolvimento da aplicação web. Na seção 4 apresentam-se os resultados e discussões, e uma avaliação inicial da aplicação após ter sido usada por um grupo de estudantes em sala de aula. Finalmente apresentam-se as considerações finais.

\section{CIRCUITOS EQUIVALENTES DO TRANSFORMADOR}

Nesta seção descrevem-se de forma sucinta os ensaios que são realizados para a determinação de parâmetros junto com as equações necessárias para o cálculo e os cinco circuitos equivalentes do transformador.

\subsection{Descrição dos ensaios clássicos}

De acordo com Chapman (2013) devem ser realizados dois ensaios para determinar os parâmetros do transformador, estes ensaios consideram o funcionamento do mesmo em condições extremas, isto é, a vazio e em curto circuito. Ditos ensaios podem ser realizados em qualquer lado do transformador, mas geralmente o ensaio de curto circuito se realiza no lado de maior tensão (portanto menor corrente) e o ensaio a vazio se realiza no lado de menor tensão (portanto maior corrente).

Conforme o lado do transformador em que foram realizadas as medições, os resultados das equações necessárias para a obtenção de cada parâmetro deverão ser modificados multiplicando ou dividindo pela relação de transformação (a). Na Tabela 1 apresentam-se as equações dos parâmetros do transformador, os índices ca representam as grandezas elétricas obtidas do ensaio a vazio e os índices cc representam as grandezas elétricas obtidas do ensaio de curto circuito.

Tabela 1 - Parâmetros do transformador monofásico.

\begin{tabular}{c|c|c}
\hline $\begin{array}{c}\text { Parâmetro obtido de } \\
\text { cada ensaio }\end{array}$ & Ensaio a vazio & Ensaio de curto circuito \\
& $\left|Z_{\varphi}\right|=\frac{V_{c a}}{I_{c a}}$ & $\left|Z_{e q}\right|=\frac{V_{c c}}{I_{c c}}$ \\
$R_{c}=\frac{V_{c a}^{2}}{P_{c a}}$ & $R_{e q}=R_{c c}=\frac{P_{c c}}{I_{c c}^{2}}$ \\
\hline Perdas características & $X_{m}=\frac{1}{\sqrt{\left(\frac{1}{Z_{\varphi}}\right)^{2}-\left(\frac{1}{R_{c}}\right)^{2}}}$ & $X_{e q}=\sqrt{\left|Z_{c c}\right|^{2}-R_{c c}^{2}}$ \\
& Pnúcleo $=\frac{V c a^{2}}{R c}$ & Pcobre $=I c c^{2} R e q$ \\
\hline
\end{tabular}

Fonte: Adaptado de Chapman (2013) 


\subsection{Circuitos equivalentes}

Os circuitos equivalentes do transformador estudados neste trabalho são representados nas figuras, sendo eles o circuito equivalente completo (Figura 1), circuito L referido ao lado primário (Figura 2), circuito $L$ referido ao lado secundário (Figura 3 ), circuito T referido ao lado primário (Figura 4), circuito T referido ao lado secundário (Figura 5).

Observa-se uma semelhança gráfica entre a Figura 2 e Figura 3, mas os valores dos parâmetros são diferentes. Essa mesma situação ocorre para a Figura 4 a Figura 5. A definição correta dos parâmetros é importante para construir o circuito equivalente completo do transformador apresentado na Figura 1.

Figura 1 - Circuitos equivalentes do transformador monofásico.

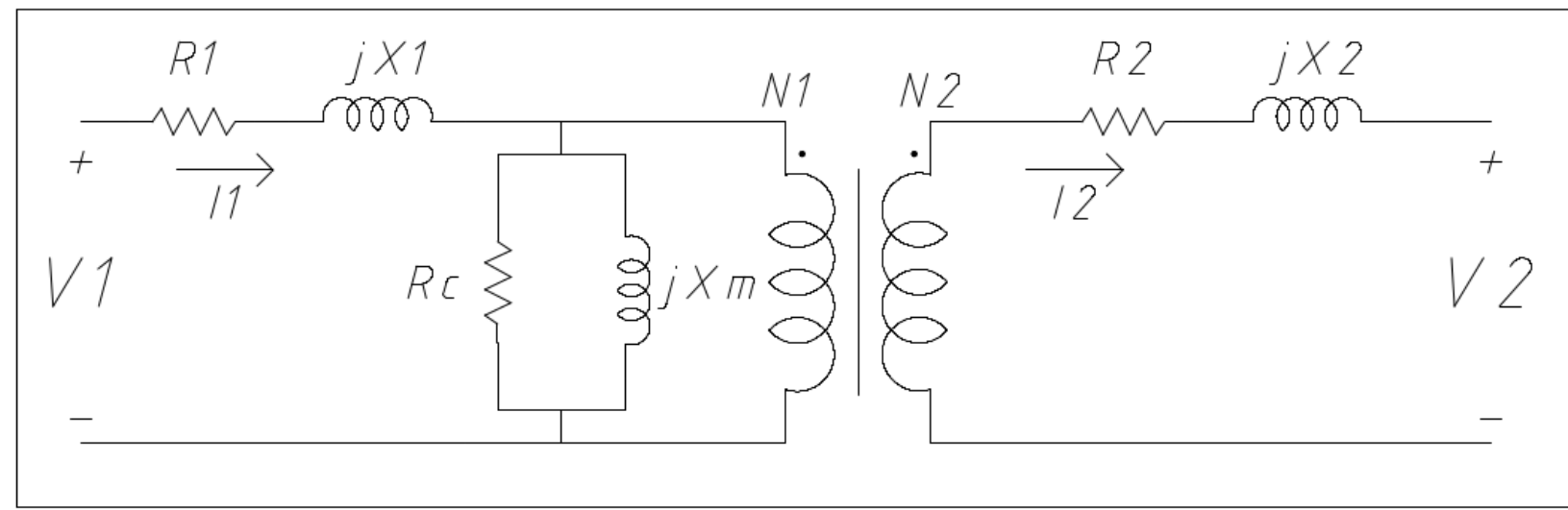

Fonte: Elaboração própria

Figura 2 - Circuito $L$ referido ao lado primário do transformador monofásico.

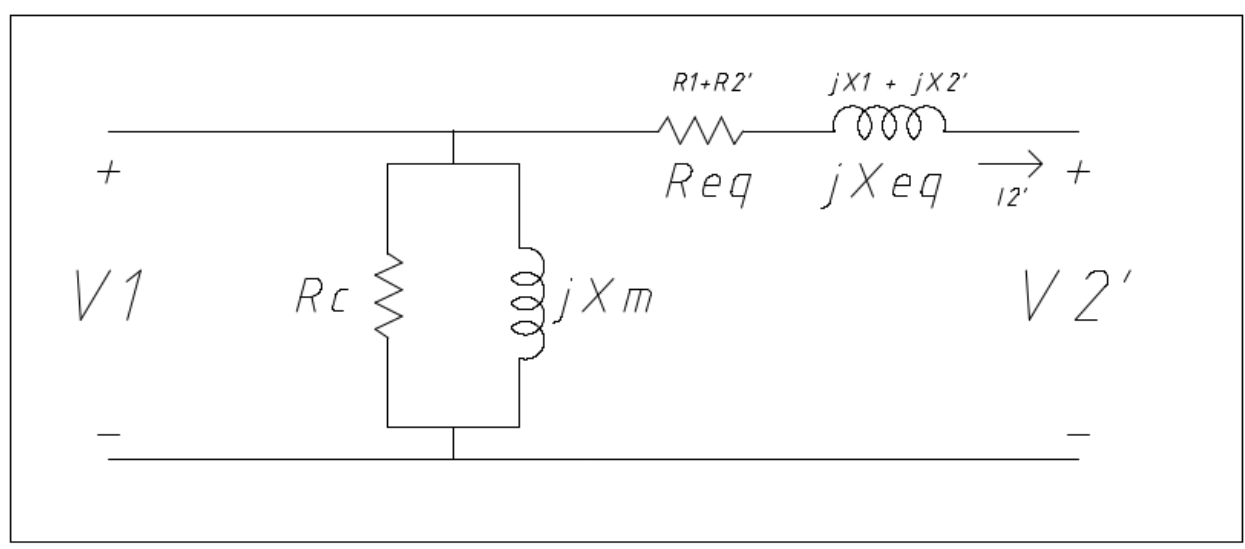

Fonte: Elaboração própria 
Figura 3 - Circuito $L$ referido ao lado secundário do transformador monofásico.

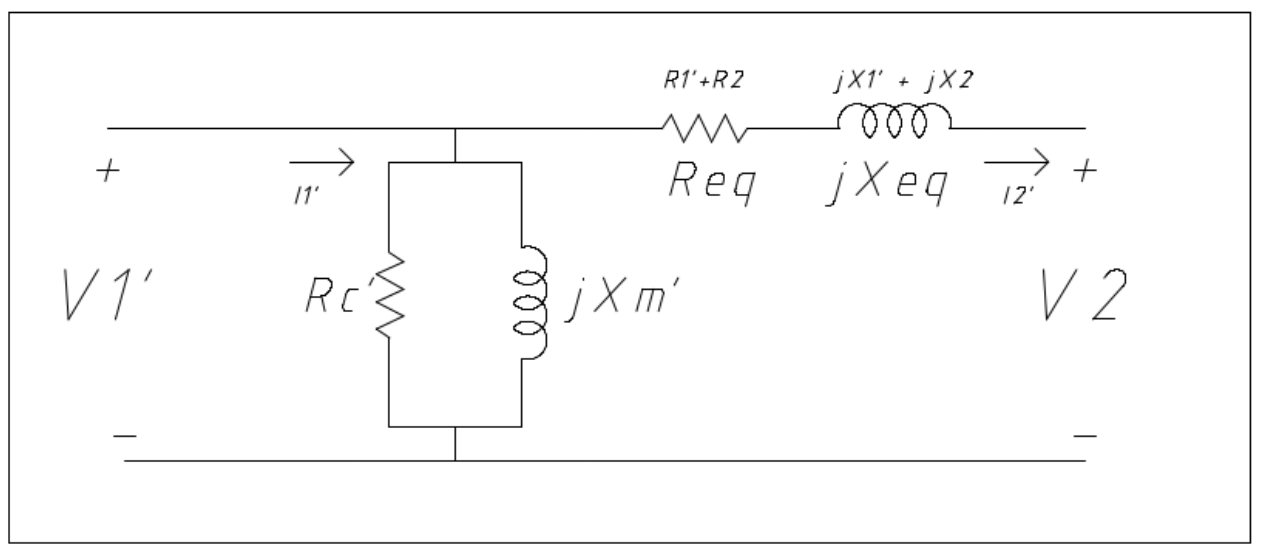

Fonte: Elaboração própria

Figura 4 - Circuito T referido ao primário do transformador monofásico.

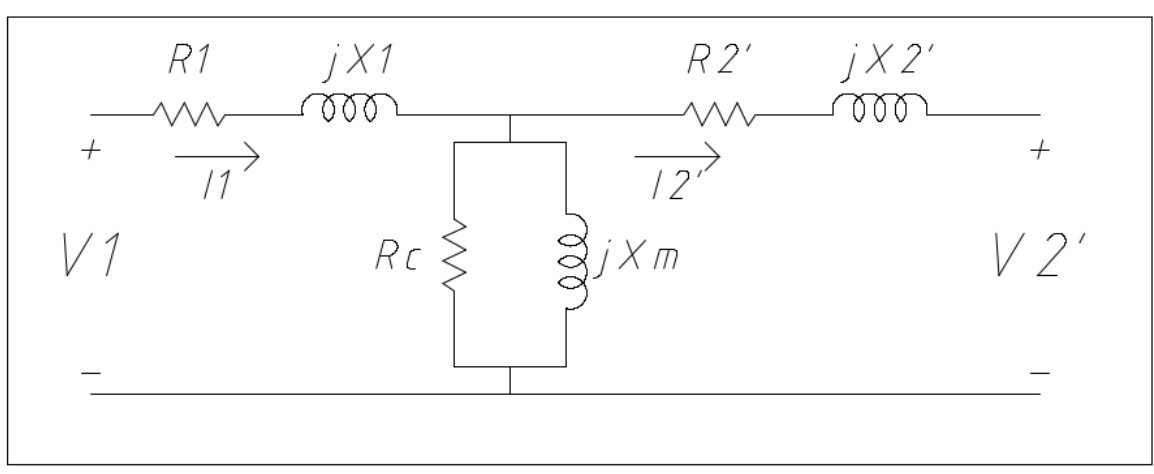

Fonte: Elaboração própria

Figura 5 - Circuito T referido ao secundário do transformador monofásico.

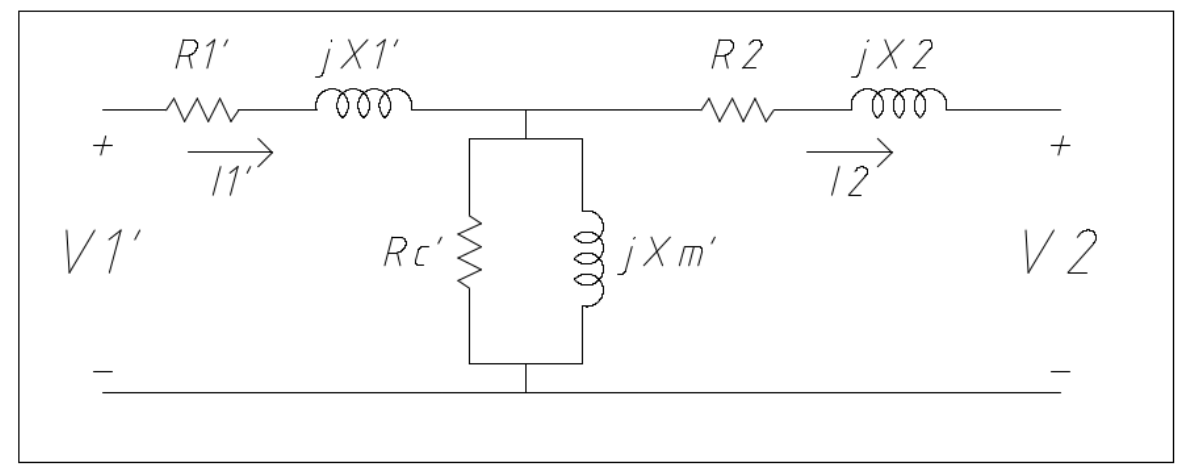

Fonte: Elaboração própria 


\section{DESENVOLVIMENTO DA APLICAÇÃO WEB}

No trabalho Nafea et al. (2019) observa-se a necessidade de elaboração de OAs de acordo com o perfil do estudante, como complemento em Yu et al. (2014) que apresenta um aprimoramento das estratégia de uso de OAs, estruturando o que é conhecido como Célula de Aprendizagem, que tem como principal contribuição o fato de garantir que o OA seja acessível de forma ubíqua sendo portanto requisito que ele possa ser acessado desde qualquer tipo de dispositivo. Dessa forma escolheu-se aprimorar o OA apresentado por Herrera et al. (2020), desenvolvendo um website garantindo dessa forma o acesso desde celulares, tablets e computadores.

As aplicações web são amplamente usadas nas diversas áreas, comerciais, entretenimento e também é claro atingem a área de tecnologia e educação. Em Lee et al. (2016) observa-se um estudo detalhado sobre aplicações web usando Open Sources (OSS), apresentando trabalhos com diversos focos.

O aplicativo desenvolvido está hospedado em uma plataforma chamada Vercel, que permite que diretórios do Github com páginas estáticas sejam postadas online de forma gratuita. Logicamente, todo o código do aplicativo está disponível em um repositório público, sendo assim Open Source, que pode ser acessado por meio deste link: $<$ https://github.com/natrodrigo/resistencia-interna-trafo>.

Para desenvolver o aplicativo, foram utilizadas as seguintes linguagens de programação: HTML para criar a estrutura das páginas, CSS com o framework Bootstrap para criar a parte visual e JavaScript para validação de dados, cálculos e interações com o usuário.

A estrutura básica do programa é representada na Figura 6 , e descreve-se nas seções 3.1 e 3.2. As seções 3.3 e 3.4 apresentam alguns detalhes considerados importantes dentro da estrutura utilizada.

Figura 6 - Estrutura do algoritmo de programação

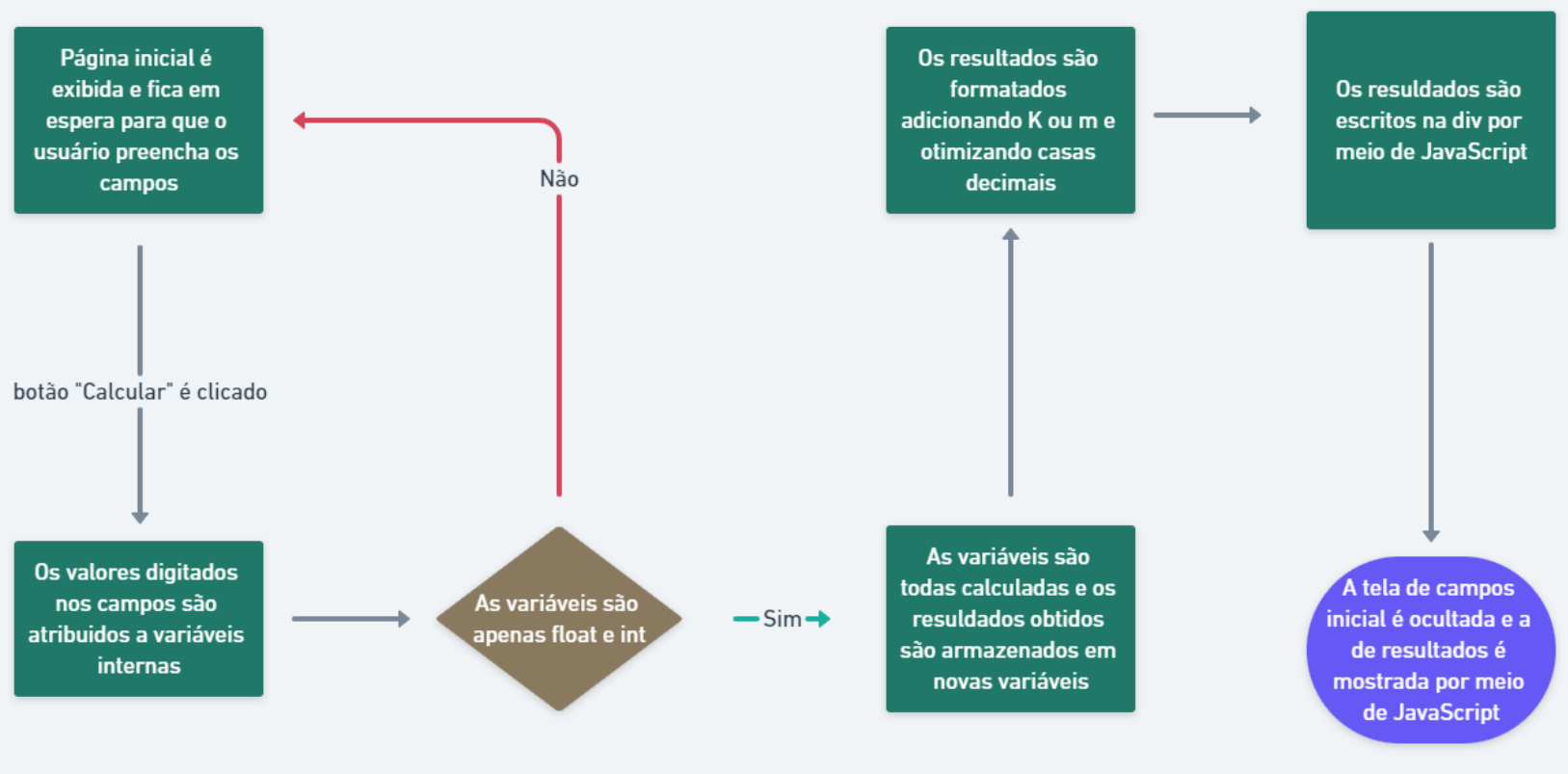

Fonte: Elaboração própria 


\subsection{Página inicial}

É onde o usuário digita os valores medidos nos ensaios (Vca, Ica, Pca, Vcc, Icc, Pcc, Vs e Vp) em campos específicos para cada medição, além do local em que foram realizadas cada uma das medições: primário ou secundário. Uma validação criada em JavaScript verifica se a entrada do usuário consiste apenas em números inteiros ou do tipo float. Caso a validação identifique que um dos campos está vazio ou não é um número, um alerta do Bootstrap em vermelho será exibido próximo ao botão "Calcular" com a mensagem: "Os campos não podem estar vazios, conter letras e os valores não podem ser iguais ou menor que zero.".

\subsection{Página de resultados}

Assim que o usuário clica no botão calcular, o valor de cada campo preenchido é atribuído à uma variável dentro do JavaScript. Cada um deles é validado, verificando se há letras, se estão vazios, se valem 0 e etc. Apenas números inteiros e do tipo float são aceitos. Os valores então são calculados para obter os resultados utilizando o sistema matemático do próprio JavaScript, e em seguida a <div> que contém todos os formulários é ocultada, e a <div> que contém as variáveis de resultados é exibida na tela com os resultados calculados.

\subsection{Página de "dúvidas"}

É uma página simples com uma explicação básica das perdas do transformador e de como o aplicativo funciona, de quais valores são calculados e qual a finalidade deles. Além disso, nessa página é também exposto todo o cálculo matemático interno do aplicativo.

\subsection{Botões de valores padrão}

Próximo ao botão calcular existem botões que ao serem clicados mostram uma simulação do funcionamento do programa com algumas variáveis pré-definidas. Cada um deles simula o funcionamento em alguma condição: teste CA (circuito aberto) e CC (curtocircuito) no primário, CA no primário e CC no secundário, CA no secundário e CC no primário e finalmente $\mathrm{CA}$ e $\mathrm{CC}$ no secundário.

\section{RESULTADOS}

Nesta seção apresentam-se os resultados obtidos, isto é, a aplicação web e uma avaliação dela por usuários. A avaliação considera uma comparação desta aplicação com o OA apresentado em Herrera et al. (2020)

\subsection{Resultado da aplicação web}

A aplicação web pode ser acessada em https://resistencia-interna-trafo.vercel.app/. $\mathrm{Na}$ Figura 7, observa-se a interface inicial onde é possível inserir os valores medidos:

Vca: Tensão (em V) medida no teste de circuito aberto; Ica: Corrente (em A) medida no teste de circuito aberto; 
Pca: Potência (em W) medida com wattímetro no teste de circuito aberto;

Vcc: Tensão (em V) medida no teste de curto-circuito;

Icc: Corrente (em A) medida no teste de curto-circuito;

Pcc: Potência (em W) medida com wattímetro no teste de curto-circuito;

E os valores nominais do transformador:

Vp: Tensão (em V) nominal do primário;

Vs: Tensão (em V) nominal do secundário;

Além de indicar se as respectivas medições foram feitas no primário ou secundário.

Figura 7 - Imagem da aplicação web para

determinação de parâmetros do transformador

Dados do ensaio de Circuito aberto

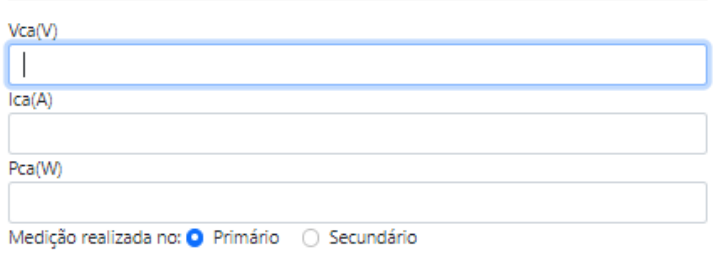

Dados do ensaio de Curto circuito

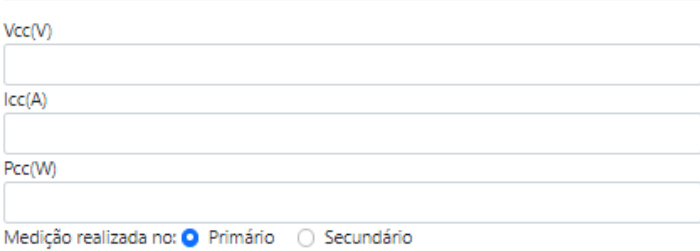

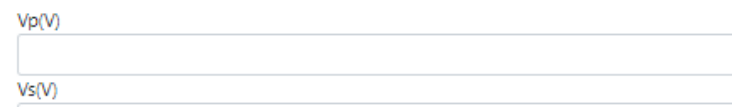

Após o usuário inserir os valores e clicar no botão calcular, ele observará os resultados como ilustrado na Figura 8. Nela pode ser observar os itens descritos na seção 2.2 , isto é, o circuito equivalente do transformador monofásico, o circuito $\mathrm{T}$ referido ao primário, circuito $T$ referido ao secundário, circuito $L$ referido ao primário e o circuito $L$ referido ao secundário. Cada um deles pode ser visto com os respectivos resultados dos cálculos das variáveis por meio dos botões de navegação destacados em vermelho na Figura 8. No início da página é possível ver quais valores foram digitados pelo usuário nos campos da página anterior, e no fim da página há um link para a página de dúvidas já citada anteriormente, além de um link para retornar à página inicial para realização de uma nova simulação. 
Figura 8 - Imagem da tela de resultados

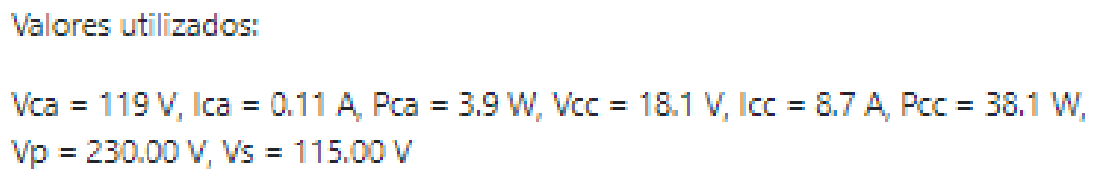

\section{Real Cir. T Primário Cir. T Secundário Cir. L Primário Cir. L Secundário}

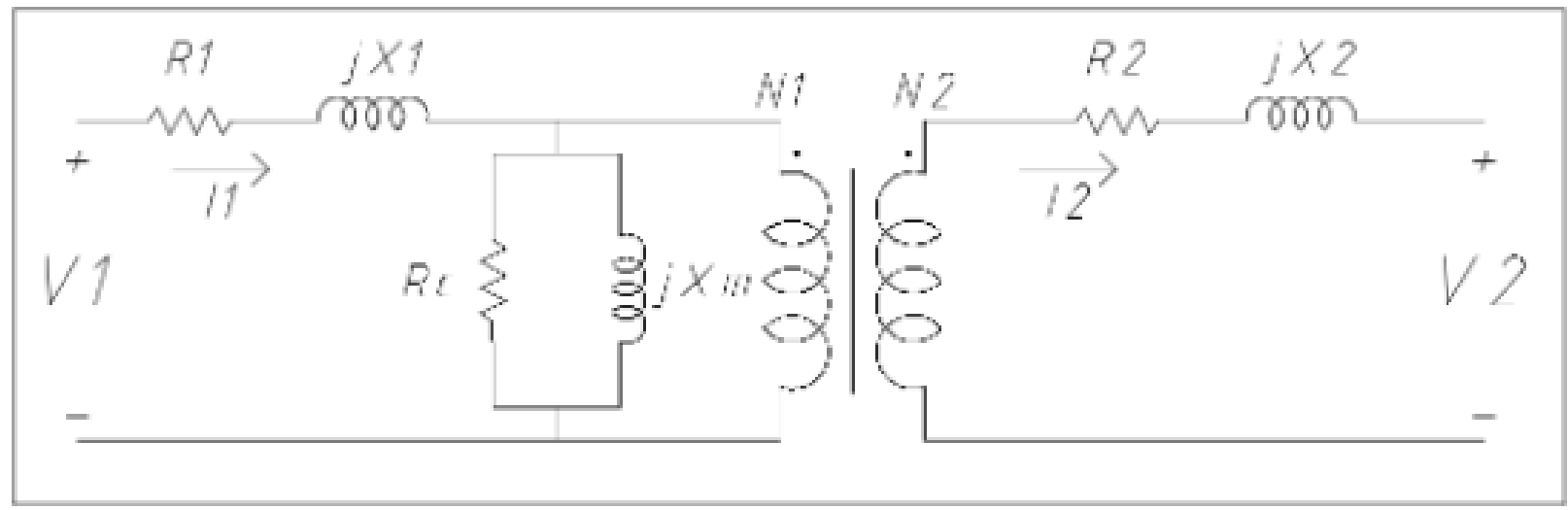

$$
\begin{aligned}
& \mathrm{R}_{1}=1.01 \Omega \\
& \mathrm{jX}=4.04 \Omega \\
& \mathrm{R}_{\mathrm{c}}=3.63 \mathrm{k} \Omega \\
& \mathrm{jX}=1.13 \mathrm{k} \Omega \\
& \mathrm{R}_{2}=251.68 \mathrm{~m} \Omega \\
& \mathrm{jX}=1.01 \Omega
\end{aligned}
$$

Perdas no núcleo $=3.90 \mathrm{~W}$

Perdas no cobre $=152,40 \mathrm{~W}$

Clique aqui para voltar à página inicial.

Dúvidas sobre como usar o aplicativo? Clique aqui. Desenvolvido por Natã Rodrigo. Visite meu perfil no Github. Fonte: Elaboração própria

\subsection{Avaliação da usabilidade}

A aplicação foi avaliada por uma turma de estudantes que após a explicação e uso preencheram um formulário anônimo, no qual foi possível avaliar a usabilidade. Não foi possível verificar os objetivos de aprendizagem, pois os estudantes usaram o OA proposto por Herrera et al. (2020), quando estavam cursando a disciplina, mas a aplicação website foi usada por eles no semestre subsequente.

Sobre o dispositivo usado para o acesso, $80 \%$ dos estudantes usaram o website no computador e o $20 \%$ no celular, nenhum estudante utilizou em tablet. Na Figuras 9 e Figura 10 apresentam-se as respostas a perguntas considerando o OA em Microsoft Excel proposto por Herrera et al. (2020), e o website proposto neste trabalho. As questões foram 
elaboradas em linguagem amigável e mais comum para o tipo de usuários (faixa etária de 22 a 26 anos conforme informado no questionário).

Na Figura 11, apresenta-se as respostas dos estudantes comparando os dois OAs. Além desse questionamento, eles responderam com comentários que ressaltaram a facilidade de uso, vantagem de não precisar instalar um programa extra e poder usá-lo em dispositivos móveis.

Figura 9 - Respostas à pergunta: Como você avalia a apresentação e aparência do objeto de

aprendizagem? a) OA Microsoft Excel, b) OA website,

c) Legendas

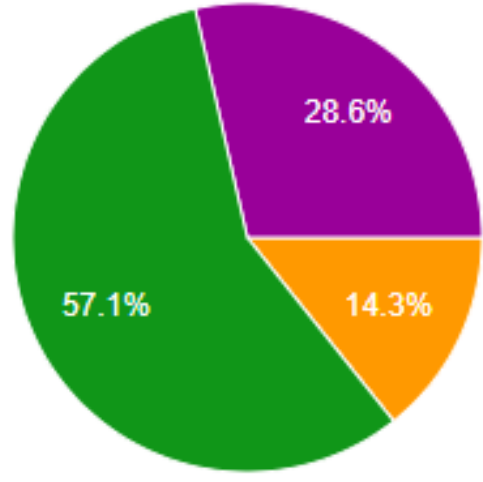

a)

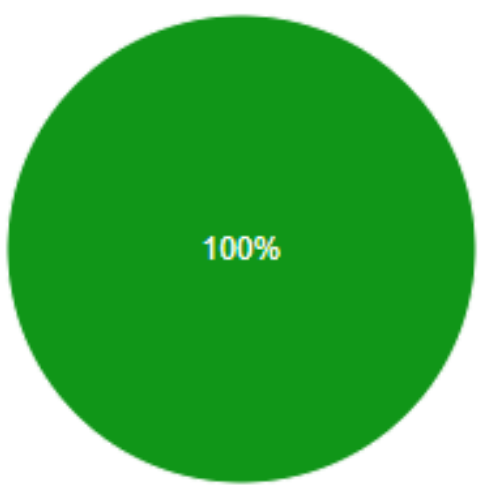

b)
Péssimo, achei feio e terrivel!

Razoável, é feio mas não terrivel.

Aceitável, bonitinho (feio arrumado).

Bom, está no caminho certo.

Ótimo, é a oitava maravilha do mundo.

Fonte: Elaboração própria

Figura 10 - Respostas à pergunta: Como você avalia a funcionalidade do objeto de aprendizagem? a) OA

Microsoft Excel, b) OA website, c) Legendas

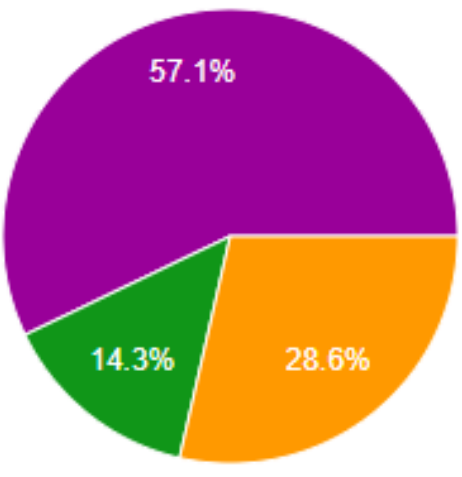

a)

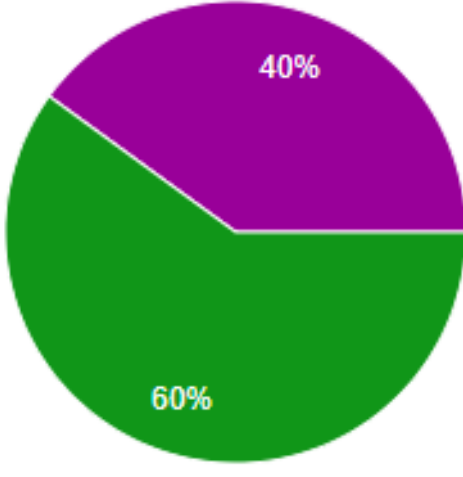

b)
Péssimo, não consegui chegar a tela de cálculo.

Razoável, achei o botão iniciar mas não funciona.

Aceitável, abri a tela de cálculos mas não é muito intuitiva.

Bom, abri a tela de cálculos e é bem intuitiva.

Ótimo, muito intuitiva, facilitou a minha vida.

\section{c)}

Fonte: Elaboração própria

Figura 11 - Respostas à pergunta: Quando comparado com o APP do Excel (usado ano passado 
em sala de aula) o que você considera? a) OA website, b) Legendas

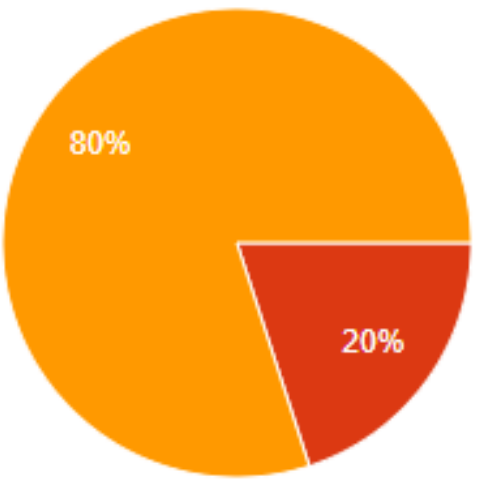

a)
Prefiro o do excel mesmo precisando de ter a versão office paga

Acho muito vantajoso não precisar de outro programa ó abrir o link e pá

Acho vantajoso porque posso usar no $\mathrm{PC}$ e no celular na boa

b)

Fonte: Elaboração própria

\section{CONSIDERAÇÕES FINAIS}

Após a elaboração da aplicação web verificaram-se alguns assuntos que devem ser aprimorados neste estudo, os quais são elencados a seguir:

Quanto à etapa de desenvolvimento, verificamos a necessidade de estudo de algumas metodologias para desenvolvimento como UWE (UML Based Web Engineering) e OOHDM (Object Oriented Hypermedia Design Methodology), citadas em Molina-Ríos; Pedreira-Souto (2020).

Quanto à avaliação de usabilidade, os autores visam continuar avaliando a aplicação web com turmas de estudantes que estudam o tema transformadores.

Quanto ao objetivo pedagógico da aplicação web mediante a avaliação dos estudantes e a visão docente considera-se que ele cumpre com as principais características desejadas de um OA, isto é, ele pode ser reutilizado em qualquer contexto de aprendizagem que contenha como conteúdo o transformador; ele é adaptável a qualquer ambiente de ensino, seja EaD, ERE ou presencial; conta com durabilidade e interoperabilidade sem risco de não poder operar por falta de atualização; e conta com metadados. Como futuro aprimoramento é necessário pensar na acessibilidade.

Finalmente, quanto ao objetivo principal da aplicação web se faz necessário elaborar e aplicar uma estrutura de utilização por docentes e alunos e forma diferenciada. Focando na visão docente, considera-se que, caso ausência de laboratório o professor pode usar este OA em complemento com os trabalhos de Ayasun; Nwankpa (2006) ou Koochaki (2015), ele deverá obter os parâmetros do transformador na aplicação web, e inseri-los nas simulações do MATLAB®/Simulink para poder fazer uma demonstração aos estudantes sobre como funcionam os ensaios clássicos; em caso de ter acesso a laboratório, o docente pode usar o OA para verificação dos resultados obtidos nos ensaios. Os estudantes, por outra parte, podem usar este OA para verificar se conseguiram realizar os cálculos corretamente, tendo a opção rápida de todos os circuitos o estudante pode facilmente verificar onde errou caso não tenha obtido um resultado adequado.

\section{Agradecimentos}

Ao programa de iniciação científica da faculdade 


\section{REFERÊNCIAS}

ACADEMY. Magnet. Transformers: interactive tutorials. Disponível em: $<$ https://nationalmaglab.org/education/magnet-academy/watchplay/interactive/transformers $>$. .

AYASUN, S.; NWANKPA, C. O. Transformer tests using MATLAB/Simulink and their integration into undergraduate electric machinery courses. Computer Applications in Engineering Education, v. 14, n. 2, p. 142-150, 2006. Disponível em: <http://doi.wiley.com/10.1002/cae.20077>. .

BRAGA, J. ORG. Objetos de Aprendizagem: Introdução e fundamentos. 2014.

CHAPMAN, S. Fundamentos de Máquinas Elétricas. 2013.

HERRERA, V.; EHARA, G.; NAKAYA, R.; DE SOUZA, A.; MOREIRA, Y. Objeto de aprendizagem para suporte ao aprendizado da determinação de parâmetros de transformadores monofásicos. Anais COBENGE 2020 - ISSN 2175 - 957X.. p.10, 2020.

HOLDINGS, M. G. H. G. E. Virtual Lab: how a transformer works?. Disponível em: <http://www.glencoe.com/sites/common_assets/science/virtual_labs/E15/E15.html>. .

KOOCHAKI, A. Teaching Calculation of Transformer Equivalent Circuit Parameters using MATLAB/Simulink for Undergraduate Electric Machinery Courses. Indian Journal of Science and Technology, v. 8, n. 17, 2015. Disponível em: $<$ https://indjst.org/articles/teaching-calculation-of-transformer-equivalent-circuit-

parameters-using-matlabsimulink-for-undergraduate-electric-machinery-courses $>$. .

LEE, T. K.; WEI, K. T.; ABD GHANI, A. A. Systematic literature review on effort estimation for Open Sources (OSS) web application development. 2016 Future Technologies Conference (FTC). Anais... . p.1158-1167, 2016. IEEE. Disponível em: $<$ https://ieeexplore.ieee.org/document/7821748/>. .

LOPES, T. DA S.; AGUIAR, V. DE P.; FREITAS DE MOURA, A. Simulações por elementos finitos aplicadas aos ensaios de um transformador em pré-aula de prática laboratorial. Anais COBENGE 2020 - ISSN 2175 - 957X. Anais... . p.10, 2020.

MOLINA-RÍOS, J.; PEDREIRA-SOUTO, N. Comparison of development methodologies in web applications. Information and Software Technology, v. 119, p. 106238, 2020. Disponível em: <https://linkinghub.elsevier.com/retrieve/pii/S0950584919302551>. .

NAFEA, S. M.; SIEWE, F.; HE, Y. A Novel Algorithm for Course Learning Object Recommendation Based on Student Learning Styles. 2019 International Conference on Innovative Trends in Computer Engineering (ITCE). Anais... . p.192-201, 2019. IEEE. Disponível em: <https://ieeexplore.ieee.org/document/8646355/>. .

YU, S.; YANG, X.; CHENG, G.; WANG, M. From Learning Object to Learning Cell: A Resource Organization Model for Ubiquitous Learning. Journal of Educational Technology \& Society, v. 18, n. 2, p. 206-224, 2014.

\section{WEB APPLICATION TO OBTAIN PARAMETERS OF THE EQUIVALENT CIRCUIT OF SINGLE-PHASE TRANSFORMERS}

Abstract: This work aimed to develop a web application to calculate resistances and reactances values of the equivalent circuit of the single-phase transformer. This web 
application, characterized as a learning object, was developed in HTML, CSS and JavaScript, presenting numerical and graphic results for five types of equivalent transformer circuit. This OA emerged as an improvement proposal to a similar OA elaborated in Microsoft Excel which presented as the main disadvantage the needing of a computer with Microsoft Excel program. In addition, to improving the durability and interoperability characteristics of the initial OA, it was found that the new interface was more user friendly. Both OAs were applied to the same classroom, in different academic periods, for usability evaluation, but the pedagogical objectives achievement still needs to be verified. Finally, suggestions for using this $O A$ are presented in a different way for teachers and students.

Keywords: Web application, Learning Object, Single-phase transformer, Equivalent circuit 